Journal of Universal Mathematics

Vol.3 No.1 PP.11-20 (2020)

ISSN-2618-5660

\title{
AN ALGORITHM FOR THE SOLUTION OF FUZZY FRACTIONAL DIFFERENTIAL EQUATION
}

\author{
A.HARIR, S. MELLIANI, AND L.S.CHADLI
}

\begin{abstract}
RÉSumÉ. In this paper the variational iteration method is used to compute the solution for the fuzzy fractional order partial differential equations with fuzzy coefficients, fuzzy initial values and fuzzy forcing functions. We propose an algorithm based on $\alpha$-cut of a fuzzy set. Finally we present some examples by using our proposed algorithm.
\end{abstract}

\section{INTRODUCTION}

The successful application of fractional differential equations FDEs in modeling such as viscoelastic material [19], control [9], signal processing [?] and etc., has attracted lots of attention not only in mathematics researches, but also in other disciplines. Just as the application of fuzzy logic in differential equations of integer order has been used as an effective tool for considering uncertainty in modeling the processes, fuzzy fractional differential equations FFDEs can also offer a more comprehensive account of the process or phenomenon. This has recently captured much attention in FFDEs. As the derivative of a function is defined in the sense of Riemann-Liouville, GrÂ $\frac{1}{4}$ nwald-Letnikov or Caputo in fractional calculus, the used derivative is to be specified and defined in FFDEs as well. FFDEs are examined in [4, 1]. The Caputo fuzzy fractional derivatives are applied here [3]. The variational iteration method have been used for solving the fuzzy fractional differential equations.

The variational iteration method [12, 13, 11] has been extensively worked out for many years by numerous authors. In this method, the equations are initially approximated with possible unknowns. A correction functional is established by the general Lagrange multiplier which can be identified optimally via the variational theory. The method provides rapidly the convergent successive approximations of the exact solution.

In brief, this paper we propose a new algorithm based on analysis of crisp solution solving by the variational iteration method. We establish a synthesis of crisp solution of fuzzy fractional differential equation and the method proposed in [17] to solve fuzzy fractional differential equation.

This paper is organized as follows : In Section 2, by defining the notation where we will use in the paper and in Sections 3, the VIM are illustrated, in section 4, the algorithm is presented for fuzzy fractional equation, in Section 5, some examples are illustrated, the conclusions are then given in the final Section 6 .

\section{Preliminaries}

We place a bar over a capital letter to denote a fuzzy number of An $\alpha$-cut of $\bar{A}$ is always a closed and bounded interval that written $\bar{A}[\alpha]$, is defined as $\left\{t \mid \mu_{\bar{A}}(t) \geq \alpha\right\}$ for $0<\alpha<1$. We separately specify $\bar{A}[0]$ as the closure of the union of all the $\bar{A}[\alpha]$ for $0<\alpha \leq 1$

2000 Mathematics Subject Classification. algorithm for the solution; fuzzy fractional differential equation.

Key words and phrases. fuzzy number, the fuzzy fractional differential equation, Variational iteration method. 
Definition 2.1. [8] Let $\mathbb{R}_{\mathcal{F}}=\{\bar{A} \mid \bar{A}: \mathbb{R} \rightarrow[0,1]$, satisfies $(1)-(4)\}:$

(1) $\forall \bar{A} \in \mathbb{R}_{\mathcal{F}}, \bar{A}$ is normal.

(2) $\forall \bar{A} \in \mathbb{R}_{\mathcal{F}}, \bar{A}$ is a fuzzy convex set.

(3) $\forall \bar{A} \in \mathbb{R}_{\mathcal{F}}, \bar{A}$ is upper semi-continuous on $\mathbb{R}$.

(4) $\bar{A}[0]$ is a compact set.

Then $\mathbb{R}_{\mathcal{F}}$ is called fuzzy number space and $\forall \bar{A} \in \mathbb{R}_{\mathcal{F}}, \bar{A}$ is called a fuzzy number.

Definition 2.2. [8, [18] We represent an arbitrary fuzzy number by an ordered pair of functions $\bar{A}[\alpha]=$ $\left[A_{1}(\alpha), A_{2}(\alpha)\right], \quad \alpha \in[0,1]$, which satisfy the following requirements :

(1) $A_{1}(\alpha)$ is a nondecreasing function over $[0,1]$,

(2) $A_{2}(\alpha)$ is a nonincreasing function on $[0,1]$

(3) $A_{1}(\alpha)$ and $A_{2}(\alpha)$ are bounded left continuous on $(0,1]$, and right continuous at $\alpha=0$, and

(4) $A_{1}(\alpha) \leq A_{2}(\alpha)$, for $0 \leq \alpha \leq 1$

Definition 2.3. For arbitrary fuzzy numbers $\bar{A}[\alpha]=\left[a_{1}(\alpha), a_{2}(\alpha)\right]$ and $\bar{B}[\alpha]=\left[b_{1}(\alpha), b_{2}(\alpha)\right]$ we have algebraic operations as follows :

$$
(\bar{A}+\bar{B})[\alpha]=\left[a_{1}(\alpha)+b_{1}(\alpha), a_{2}(\alpha)+b_{2}(\alpha)\right] \quad k \bar{A}[\alpha]= \begin{cases}{\left[k a_{1}(\alpha), k a_{2}(\alpha)\right]} & k \geq 0 \\ {\left[k a_{2}(\alpha), k a_{1}(\alpha)\right]} & k<0\end{cases}
$$

Let $u:(0, a) \rightarrow \mathbb{R}_{\mathcal{F}}$ be a fuzzy function. We denote

$$
[u(t)]^{\alpha}=\left[u_{1}^{\alpha}(t), u_{2}^{\alpha}(t)\right], \quad t \in T, \alpha \in[0,1]
$$

We adopt the general definition of a fuzzy number given in [10].

Definition 2.4. 2] Let $u \in C\left((0, a], \mathbb{R}_{\mathcal{F}}\right) \cap L^{1}\left((0, a), \mathbb{R}_{\mathcal{F}}\right)$. Define the fuzzy fractional primitive of order $q>0$ of $u$,

$$
\left[I^{q} u(t)\right]^{\alpha}=\left[\frac{1}{\Gamma(q)} \int_{a}^{t}(t-s)^{q-1} u(s) d s\right]^{\alpha}, \quad t \in(0, a)
$$

Definition 2.5. 3] Let $u \in C^{1+k}\left([0, a], \mathbb{R}_{\mathcal{F}}\right) \cap L^{1}\left([0, a], \mathbb{R}_{\mathcal{F}}\right)$ be a given function such that $[u]^{\alpha}=\left[u_{1}^{\alpha}, u_{2}^{\alpha}\right]$ for all $t \in(0, a)$ and $\alpha \in[0,1]$ the fuzzy fractional differential operator in the Caputo sense is defined

$$
\left[D_{c}^{q} u(t)\right]^{\alpha}=\left[\frac{1}{\Gamma(1+k-q)} \int_{0}^{t} D^{1+k}(t-s)^{k-q} u(s) d s\right]^{\alpha} .
$$

for all $t \in(0, a)$ and $\alpha \in[0,1]$

Definition 2.6. Fractional derivative of compounded functions [16] is defined as

$$
d^{q} u \cong \Gamma(1+q) d u, \quad 0<q<1
$$

The definition of the fractional integral in Eq. 2.2. is equivalent to Lemma 4.1 of [16], namely,

$$
y=\int_{0}^{t} u(s)(d s)^{q}=q \int_{0}^{t}(t-s)^{q-1} u(s) d s, \quad 0<\alpha \leq 1
$$


2.1. The fractional variation iteration method. To clarify the basic ideas of VIM, we consider the following differential equation :

$$
D_{t}^{q} U(t, x)+L U(t, x)+N U(t, x)=F(t, x, k)
$$

where $D_{t}^{q}()=.\frac{\partial^{q}}{\partial t^{q}}$ is the operator defined 2.1), $q>0, L$ is a linear operator, $N$ is a nonlinear operator, $U(t, x)$ is an unknown function, and $F(t, x, k)$ is the source inhomogeneous term. According to the VIM introduced by He [15], we can construct a correction functional for Eq. 2.4. as follows :

$$
\begin{gathered}
U_{n+1}(t, x)=U_{n}(t, x)+I_{t}^{q}\left\{\lambda(t)\left(D_{t}^{q} U_{n}(t, x)+L\left(U_{n}(t, x)\right)+N \widetilde{U}_{n}(t, x)-F(t, x, k)\right)\right\} \\
\begin{array}{r}
U_{n+1}(t, x)=U_{n}(t, x)+\frac{1}{\Gamma(q)} \int_{0}^{t}(t-\tau)^{q-1}\left\{\lambda ( \tau ) \left(D_{\tau}^{q} U_{n}(\tau, x, y)\right.\right. \\
\left.\left.+L\left(U_{n}(\tau, x)\right)+N \widetilde{U}_{n}(\tau, x)-F(\tau, x, k)\right)\right\} d \tau .
\end{array}
\end{gathered}
$$

Combining Eqs. 2.3) and (2.5), we obtain a proposed correction functional

$$
\begin{aligned}
U_{n+1}(t, x)=U_{n}(t, x)+\frac{1}{\Gamma(q+1)} \int_{0}^{t}\left\{\lambda ( \tau ) \left(D_{\tau}^{q} U_{n}(\tau, x)\right.\right. & \\
& \left.\left.+L\left(U_{n}(\tau, x)\right)+N \widetilde{U}_{n}(\tau, x, y)-F(\tau, x, k)\right)\right\}(d \tau)^{q} .
\end{aligned}
$$

where $\lambda_{i}, 1 \leq i \leq 3$ are general Lagrange multipliers, which can be identified optimally via the variational theory [14, 20, and $\widetilde{U}_{n}$ is a restricted variation which means $\delta \widetilde{U}_{n}=0$. It is required first to determine the Lagrange multipliers $\lambda_{i}$ that will be identified optimally via integration by parts. The approximations $U_{n+1}, n \geq 0$, of the solution $U(t, x)$ will immediately follow upon using any selective function $U_{0}$. The initial values $U(0, x)$ is usually used for the selected zeroth approximations $U_{0}$. With the Lagrange multipliers $\lambda_{i}$ determined, then several approximation $u_{i}(t, x), i \geq 0$, can be determined. Consequently, the solution is given as

$$
U(t, x)=\lim _{n \rightarrow \infty} U_{n}(t, x)
$$

Consider the following fractional differential equation :

$$
D_{t}^{q} U(t, x)+P(x) \Phi\left(D_{x}, D_{x^{2}}\right) U(t, x)=F(t, x)
$$

According to the VIM, we construct a correction functional for (2.7) as follows

$$
U_{n+1}(t, x)=U_{n}(t, x)+\frac{1}{\Gamma(1+q)} \times \int_{0}^{t} \lambda(\tau)\left\{D_{\tau}^{q} U_{n}+P(x) \Phi\left(D_{x}, D_{x^{2}}\right) \widetilde{U}_{n}-F(s, x)\right\}(d \tau)^{q}
$$

where $n \geq 0$ and $\lambda$ is a lagrange multiplier. We now determine the Lagrange multiplier let $0<q \leq 1$

$$
\delta U_{n+1}(t, x)=\delta U_{n}(t, x)+\delta \int_{0}^{t} \lambda(\tau)\left\{D_{\tau}^{q} U_{n}+P(x) \Phi\left(D_{x}, D_{x^{2}}\right) \widetilde{U}_{n}-F(\tau, x, k)\right\}(d \tau)^{q}
$$

Therefore, the stationary conditions are :

$$
\frac{\partial^{q} \lambda(\tau)}{\partial \tau^{q}}=0 \quad \text { and } \quad 1+\left.\lambda(\tau)\right|_{\tau=t}=0
$$

So, the Lagrange multiplier is $\lambda=-1$. Submitting the results into 2.8 leads to the following iteration formula

$$
U_{n+1}(t, x)=U_{n}(t, x)-\int_{0}^{t}\left\{D_{\tau}^{q} U_{n}(\tau, x)+P(x) \Phi\left(D_{x}, D_{x^{2}}\right) \widetilde{U}_{n}(\tau, x)-F(\tau, x, k)\right\}(d \tau)^{q}
$$


Iteration formula start with initial approximation, for example $U_{0}(t, x)=U(0, x)$.

\section{A SOLUTION METHOD OF THE FUZZY FRACTIONAL DIFFERENTIAL EQUATION}

Consider the following fuzzy fractional differential equation :

$$
\left\{\begin{array}{l}
D_{t}^{q} \bar{U}(t, x)+\bar{P}(x) \Phi\left(D_{x}, D_{x^{2}}\right) \bar{U}(t, x)=\bar{F}(t, x), \\
\bar{U}(0, x)=\bar{f}(x)
\end{array}\right.
$$

Where $\bar{P}(x), \bar{f}(x), \bar{F}(t, x)$ and $\bar{U}(t, x)$ are fuzzy functions and their $\alpha$-cut sets are as follows.

$$
\begin{gathered}
{[\bar{P}(x)]^{\alpha}=\left[p_{1}(x, \alpha), p_{2}(x, \alpha)\right], \quad[\bar{F}(t, x)]^{\alpha}=\left[F_{1}(t, x, \alpha), F_{2}(t, x, \alpha)\right],} \\
{[\bar{f}(x)]^{\alpha}=\left[f_{1}(x, \alpha), f_{2}(x, \alpha)\right], \quad \text { and } \quad[\bar{U}(t, x)]^{\alpha}=\left[U_{1}(t, x, \alpha), U_{2}(t, x, \alpha)\right] .}
\end{gathered}
$$

Hence we get from 3.1 :

$$
\begin{aligned}
{\left[D_{t}^{q} U_{1}(t, x, \alpha), D_{t}^{q} U_{2}(t, x, \alpha)\right]+\left[p_{1}(x, \alpha), p_{2}(x, \alpha)\right]\left[\Phi\left(D_{x}, D_{x^{2}}\right) U_{1}(t, x, \alpha),\right.} \\
\\
\left.\Phi\left(D_{x}, D_{x^{2}}\right) U_{2}(t, x, \alpha)\right]=\left[F_{1}(t, x, \alpha), F_{2}(t, x, \alpha)\right]
\end{aligned}
$$

$\left[U_{1}(0, x, \alpha), U_{2}(0, x, \alpha)\right]=\left[f_{1}(x, \alpha), f_{2}(x, \alpha)\right]$.

The operator $\Phi\left(D_{x}, D_{x^{2}}\right)$ will be a polynomial, with constant coefficients, in $D_{x}$ and $D_{x^{2}}$ where $D_{x}\left(D_{x^{2}}\right)$ stands for the partial with respect to $x\left(x^{2}\right)$, also, $U(t, x)$ is continuous function, having continuous partial with respect to both $x$ and $x^{2}$. As we know from interval analysis [6, 7, 17]

$$
\begin{array}{r}
\min \left\{\left[D_{t}^{q} U_{1}(t, x, \alpha), D_{t}^{q} U_{2}(t, x, \alpha)\right]+\left[p_{1}(x, \alpha), p_{2}(x, \alpha)\right]\left[\Phi\left(D_{x}, D_{x^{2}}\right) U_{1}(t, x, \alpha),\right.\right. \\
\left.\left.\Phi\left(D_{x}, D_{x^{2}}\right) U_{2}(t, x, \alpha)\right]\right\}=F_{1}(t, x, \alpha),
\end{array}
$$

and

$$
\begin{array}{r}
\max \left\{\left[D_{t}^{q} U_{1}(t, x, \alpha), D_{t}^{q} U_{2}(t, x, \alpha)\right]+\left[p_{1}(x, \alpha), p_{2}(x, \alpha)\right]\left[\Phi\left(D_{x}, D_{x^{2}}\right) U_{1}(t, x, \alpha),\right.\right. \\
\left.\left.\Phi\left(D_{x}, D_{x^{2}}\right) U_{2}(t, x, \alpha)\right]\right\}=F_{2}(t, x, \alpha),
\end{array}
$$

Then we have a min, max problem. It is not an easy task to determine the result of min and max operation so we propose the following method.

(1) Solve the crisp problem by Fractional variational iteration method (FVIM).

(2) Verify the sign of the derivative $\mathcal{D} U(t, x),\left(\mathcal{D} U(t, x)=D_{t}^{q} U(t, x)\right.$ or $\left.\mathcal{D} U(t, x)=\Phi\left(D_{x}, D_{x^{2}}\right) U(t, x)\right)$ of the crisp solution and precision of the domains.

(3) Translate the crisp problem in fuzzy problem (fuzzification) using $\alpha$-cut (level) set for $\bar{U}(t, x), \Phi\left(D_{x}, D_{x^{2}}\right) \bar{U}(t, x)$, $D_{t}^{q} \bar{U}(t, x)$. The following cases came out :

- $\left[\mathcal{D} U_{1}(t, x, \alpha), \mathcal{D} U_{2}(t, x, \alpha)\right]$ in case $\mathcal{D} U \geq 0$

- $\left[\mathcal{D} U_{2}(t, x, \alpha), \mathcal{D} U_{1}(t, x, \alpha)\right]$ in case $\mathcal{D} U \leq 0$

(4) substitute the $\alpha$-cut sets in fuzzy problem, and solved the $\alpha$-cut set operations of fuzzy fractional differential equation by Fractional variational iteration method (FVIM).see [5]

\section{EXAMPLES}

In this section we apply this algorithm for solving fuzzy fractional differential equation. 
Example 4.1. As the first example, for simplicity, we consider the following fuzzy fractional differential equation

$$
\left\{\begin{array}{l}
D_{t}^{q} \bar{U}(t, x)=\bar{K} x^{2}, \quad 0<q \leq 1 \\
\bar{U}(0, x)=\bar{C} x
\end{array}\right.
$$

where $\alpha$-cut set of initial values are $[\bar{K}]^{\alpha}=[5]^{\alpha}=[4+\alpha, 6-\alpha]$ and $[\bar{C}]^{\alpha}=[\overline{3}]^{\alpha}=[2+\alpha, 4-\alpha]$. The solution of the crisp problem corresponding to (4.1) using the variational iteration method Eq 2.9$), U(t, x)=3 x+5 x^{2} \frac{t^{q}}{\Gamma(1+q)}$ which is the exact solution of Eq. 4.1. . The derivative of crisp solution is $\frac{\partial^{q}}{\partial t^{q}} U(t, x)=5 x^{2}$. Therefore for any $\mathrm{t}$, $\mathrm{x}, \frac{\partial^{q}}{\partial t^{q}} U(t, x)>0$.

Then $\alpha$-cut set of $\bar{U}(t, x), \frac{\partial^{q}}{\partial t^{q}} \bar{U}(t, x)$ are $\left[U_{1}(t, x, \alpha), U_{2}(t, x, \alpha)\right],\left[\frac{\partial^{q}}{\partial t^{q}} U_{1}(t, x, \alpha), \frac{\partial^{q}}{\partial t^{q}} U_{2}(t, x, \alpha)\right]$ respectively. If we substitute these $\alpha$-cut sets in 4.1 we get the following problem.

$$
\left\{\begin{array}{l}
{\left[\frac{\partial^{q}}{\partial t^{q}} U_{1}(t, x, \alpha), \frac{\partial^{q}}{\partial t^{q}} U_{2}(t, x, \alpha)\right]=[4+\alpha, 6-\alpha] x^{2},} \\
{\left[U_{1}(0, x, \alpha), U_{2}(0, x, \alpha)\right]=[2+\alpha, 4-\alpha] x}
\end{array} .\right.
$$

and the solution for 4.2 is as follows.

$$
\left\{\begin{array}{l}
U_{1}(t, x, \alpha)=4 x^{2} \frac{t^{q}}{\Gamma(1+q)}+\alpha x^{2} \frac{t^{q}}{\Gamma(1+q)}+\alpha x+2 x, \\
U_{2}(t, x, \alpha)=6 x^{2} \frac{t^{q}}{\Gamma(1+q)}-\alpha x^{2} \frac{t^{q}}{\Gamma(1+q)}-\alpha x+4 x .
\end{array}\right.
$$

Now, in this point we must ensure that $U_{1}(t, x, \alpha) \leq U_{1}(t, x, 1)=U_{2}(t, x, 1) \leq U_{2}(t, x, \alpha)$ and $\frac{\partial^{q}}{\partial t^{q}} U_{1}(t, x, \alpha) \leq$ $\frac{\partial^{q}}{\partial t^{q}} U_{2}(t, x, \alpha), \forall \alpha \in[0,1]$. we see that $U_{1}(t, x, \alpha), U_{2}(t, x, \alpha)$ represent a fuzzy number when $x, t \geq 0$ and the order of the time-fractional derivative $\frac{\partial^{q}}{\partial t^{q}} U_{1}(t, x, \alpha)=(4+\alpha) x^{2}, \frac{\partial^{q}}{\partial t^{q}} U_{2}(t, x, \alpha)=(6-\alpha) x^{2}$ give fuzzy number. Therefore (4.3) is a fuzzy solution for (4.1) for every $t, x \geq 0$. In figure 1 solution curves of (4.1) are given.
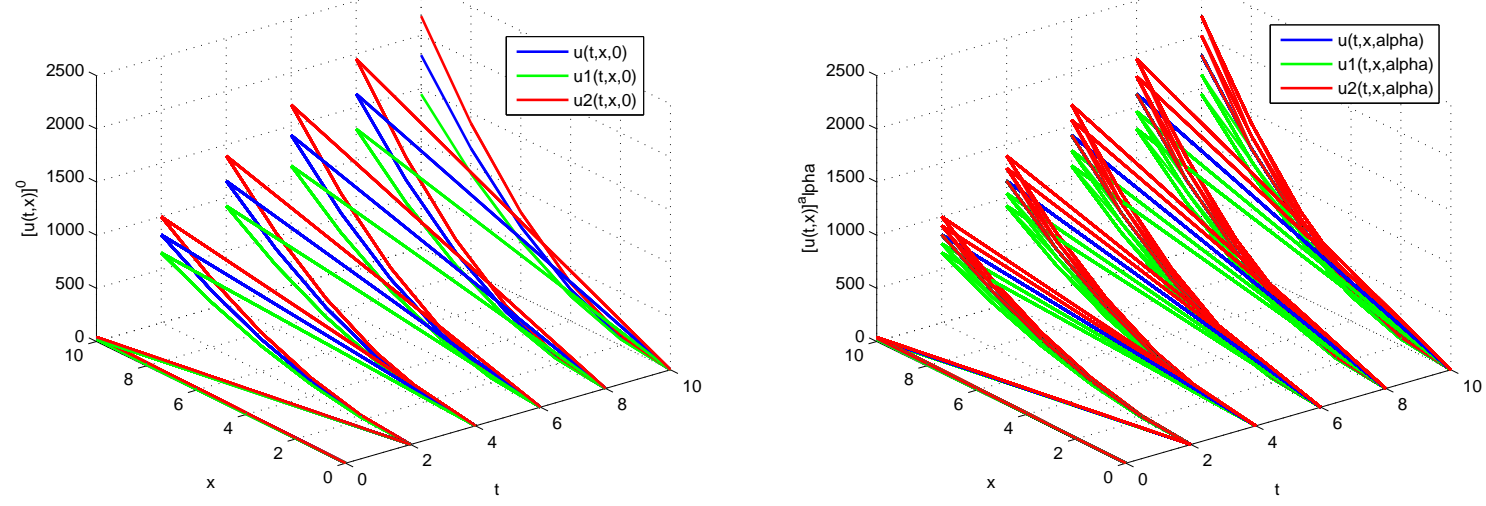

Figure 1. Fuzzy solution for $\alpha=0$ and $\alpha \in[0,1]$ to 4.1 
Example 4.2. Consider the fuzzy fractional differential equation

$$
\left\{\begin{array}{l}
D_{t}^{q} \bar{U}(t, x)+\bar{\gamma} x \frac{\partial^{2}}{\partial x^{2}} \bar{U}(t, x)=\bar{K} x t \\
\bar{U}(0, x)=\bar{C} x^{2}
\end{array}\right.
$$

where $\alpha$-cut set of initial value is $[\bar{C}]^{\alpha}=[\overline{2}]^{\alpha}=[1+\alpha, 3-\alpha]$ and $\alpha$-cut set of coefficient is $[\bar{\gamma}]^{\alpha}=[\overline{1}]^{\alpha}=[\alpha, 2-\alpha]$ and $\alpha$-cut set of forcing term is $[\bar{K}]^{\alpha}=[\overline{3}]^{\alpha}=[2+\alpha, 4-\alpha]$. Now, before solving fuzzy fractional equation let us solve crisp problem corresponding to (4.4), to analyze behavior of solution. The crisp problem is

$$
\left\{\begin{array}{l}
D_{t}^{q} U(t, x)+x \frac{\partial^{2}}{\partial x^{2}} U(t, x)=3 x t, \quad 0<q \leq 1 \\
\bar{U}(0, x)=2 x^{2} .
\end{array}\right.
$$

According to the VIM, a correct functional for 4.5) from (2.9) we can obtain the solution for (4.5) is $U(t, x)=$ $2 x^{2}-4 x \frac{t^{q}}{\Gamma(1+q)}+3 x \frac{t^{1+q}}{\Gamma(2+q)}$. The derivatives of crisp solution are $\frac{\partial^{2}}{\partial x^{2}} U(t, x)=4, \frac{\partial^{q}}{\partial t^{q}} U(t, x)=-4 x+3 x t$. So for any t,x, $\frac{\partial^{2}}{\partial x^{2}} U(t, x)>0$ and $x>0, t \geq \frac{4}{3}, \frac{\partial^{q}}{\partial t^{q}} U(t, x) \geq 0$. Hence in domain $x>0, t \geq \frac{4}{3}$ the $\alpha$-cut set of $\bar{U}(t, x)$, $\frac{\partial^{2}}{\partial x^{2}} \bar{U}(t, x), \frac{\partial^{q}}{\partial t^{q}} \bar{U}(t, x)$ are $\left[U_{1}(t, x, \alpha), U_{2}(t, x, \alpha)\right]$, $\left[\frac{\partial^{2}}{\partial x^{2}} U_{1}(t, x, \alpha), \frac{\partial^{2}}{\partial x^{2}} U_{2}(t, x, \alpha)\right],\left[\frac{\partial^{q}}{\partial t^{q}} U_{1}(t, x, \alpha), \frac{\partial^{q}}{\partial t^{q}} U_{2}(t, x, \alpha)\right]$ respectively.

Now if we substitute these $\alpha$-cut sets in (4.4) we get the following.

$$
\left\{\begin{array}{l}
{\left[\frac{\partial^{q}}{\partial t^{q}} U_{1}(t, x, \alpha), \frac{\partial^{q}}{\partial t^{q}} U_{2}(t, x, \alpha)\right]+[\alpha, 2-\alpha] x\left[\frac{\partial^{2}}{\partial x^{2}} U_{1}(t, x, \alpha), \frac{\partial^{2}}{\partial x^{2}} U_{2}(t, x, \alpha)\right]=[2+\alpha, 4-\alpha] x t,} \\
{\left[U_{1}(0, x, \alpha), U_{2}(0, x, \alpha)\right]=[1+\alpha, 3-\alpha] x^{2} .}
\end{array}\right.
$$

so the solution for (4.7) is as follows.

$$
\left\{\begin{array}{l}
U_{1}(t, x, \alpha)=(1+\alpha) x^{2}-\left(2 \alpha+2 \alpha^{2}\right) x \frac{t^{q}}{\Gamma(1+q)}+(2+\alpha) x \frac{t^{q+1}}{\Gamma(2+q)} \\
U_{2}(t, x, \alpha)=(3-\alpha) x^{2}-\left(2 \alpha^{2}-10 \alpha+12\right) x \frac{t^{q}}{\Gamma(1+q)}+(4-\alpha) x \frac{t^{q+1}}{\Gamma(2+q)} .
\end{array}\right.
$$

we see that $U_{1}(t, x, 1)-U_{1}(t, x, \alpha) \geq 0, U_{2}(t, x, \alpha)-U_{2}(t, x, 1) \geq 0$ when $x^{2}-6 x \frac{t^{q}}{\Gamma(1+q)}+x \frac{t^{1+q}}{2+q} \geq 0$ i.e $x \geq 0$ or $x \leq 6 \frac{t^{q}}{\Gamma(1+q)}-\frac{t^{1+q}}{\Gamma(2+q)}$ when $6 t-\frac{t^{2}}{2} \leq 0$ i.e $t \leq 0$ and $t \geq 12$. The second derivatives $\frac{\partial^{2}}{\partial x^{2}} U_{1}(t, x, \alpha), \frac{\partial^{2}}{\partial x^{2}} U_{2}(t, x, \alpha)$ give fuzzy number for every $\mathrm{x}$,t. The time-fractional derivative of $U_{1}(t, x, \alpha), U_{2}(t, x, \alpha)$ are $\frac{\partial^{q}}{\partial t^{q}} U_{1}(t, x, \alpha)=$ $-\left(2 \alpha+2 \alpha^{2}\right) x+(2+\alpha) x t, \frac{\partial^{q}}{\partial t^{q}} U_{2}(t, x, \alpha)=-\left(2 \alpha^{2}-10 \alpha+12\right) x+(4-\alpha) x t$ respectively and $\frac{\partial^{q}}{\partial t^{q}} U_{2}(t, x, \alpha) \geq$ $\frac{\partial^{q}}{\partial t^{q}} U_{2}(t, x, 1), \frac{\partial^{q}}{\partial t^{q}} U_{1}(t, x, 1) \geq \frac{\partial^{q}}{\partial t^{q}} U_{1}(t, x, \alpha)$ when $x>0, t \geq 8$ hence represent a fuzzy number for $x>0, t \geq 8$ for $\alpha \in[0,1]$. The solution for (4.4) given in figure 2 for $\alpha=0$ and in figure 3 for $\alpha \in[0,1]$.

Similarly we can do same process for $x>0, t \leq \frac{4}{3}$ We have found the $\alpha$-cut set of $\bar{U}(t, x), \frac{\partial^{2}}{\partial x^{2}} \bar{U}(t, x)$, $\frac{\partial^{q}}{\partial t^{q}} \bar{U}(t, x)$ as $\left[U_{1}(t, x, \alpha), U_{2}(t, x, \alpha)\right],\left[\frac{\partial^{2}}{\partial x^{2}} U_{1}(t, x, \alpha), \frac{\partial^{2}}{\partial x^{2}} U_{2}(t, x, \alpha)\right],\left[\frac{\partial^{q}}{\partial t^{q}} U_{2}(t, x, \alpha), \frac{\partial^{q}}{\partial t^{q}} U_{1}(t, x, \alpha)\right]$ respectively. If we substitute these $\alpha$-cut sets in 4.4 for $x>0, t \leq \frac{4}{3}$ we get the following

$$
\left\{\begin{array}{l}
{\left[\frac{\partial^{q}}{\partial t^{q}} U_{2}(t, x, \alpha), \frac{\partial^{q}}{\partial t^{q}} U_{1}(t, x, \alpha)\right]+[\alpha, 2-\alpha] x\left[\frac{\partial^{2}}{\partial x^{2}} U_{1}(t, x, \alpha), \frac{\partial^{2}}{\partial x^{2}} U_{2}(t, x, \alpha)\right]=[2+\alpha, 4-\alpha] x t,} \\
{\left[U_{1}(0, x, \alpha), U_{2}(0, x, \alpha)\right]=[1+\alpha, 3-\alpha] x^{2} .}
\end{array}\right.
$$

so the solution for 4.8 is as follows.

$$
\left\{\begin{array}{l}
U_{1}(t, x, \alpha)=(1+\alpha) x^{2}-\left(2 \alpha^{2}-10 \alpha+12\right) x \frac{t^{q}}{\Gamma(1+q)}+(2+\alpha) x \frac{t^{q+1}}{\Gamma(2+q)}, \\
U_{2}(t, x, \alpha)=(3-\alpha) x^{2}-\left(2 \alpha+2 \alpha^{2}\right) x \frac{t^{q}}{\Gamma(1+q)}+(4-\alpha) x \frac{t^{q+1}}{\Gamma(2+q)} .
\end{array}\right.
$$

Now, in this point we must ensure that $U_{1}(t, x, 1)-U_{1}(t, x, \alpha) \geq 0, U_{2}(t, x, \alpha) \geq U_{2}(t, x, 1)=U_{1}(t, x, 1) \geq$ $U_{1}(t, x, \alpha)$ when $x^{2}+4 x \frac{t^{q}}{\Gamma(1+q)}+x \frac{t^{1+q}}{2+q} \geq 0$ i.e $x \geq 0$ or $x \leq-4 \frac{t^{q}}{\Gamma(1+q)}-\frac{t^{1+q}}{\Gamma(2+q)}$ when $-4 t-\frac{t^{2}}{2} \leq 0$ i.e $t \geq 0$ and $t \leq-8$. The second derivatives $\frac{\partial^{2}}{\partial x^{2}} U_{1}(t, x, \alpha), \frac{\partial^{2}}{\partial x^{2}} U_{2}(t, x, \alpha)$ give fuzzy number for every $x, t$. The time-fractional derivative of $U_{1}(t, x, \alpha), U_{2}(t, x, \alpha)$ are $\frac{\partial^{q}}{\partial t^{q}} U_{1}(t, x, \alpha)=-\left(2 \alpha^{2}-10 \alpha+12\right) x+(2+\alpha) x t$, $\frac{\partial^{q}}{\partial t^{q}} U_{2}(t, x, \alpha)=-\left(2 \alpha+2 \alpha^{2}\right) x+(4-\alpha) x t$ respectively and $\frac{\partial^{q}}{\partial t^{q}} U_{2}(t, x, \alpha) \geq \frac{\partial^{q}}{\partial t^{q}} U_{1}(t, x, \alpha)$ when $x>0, t \geq-4$ 


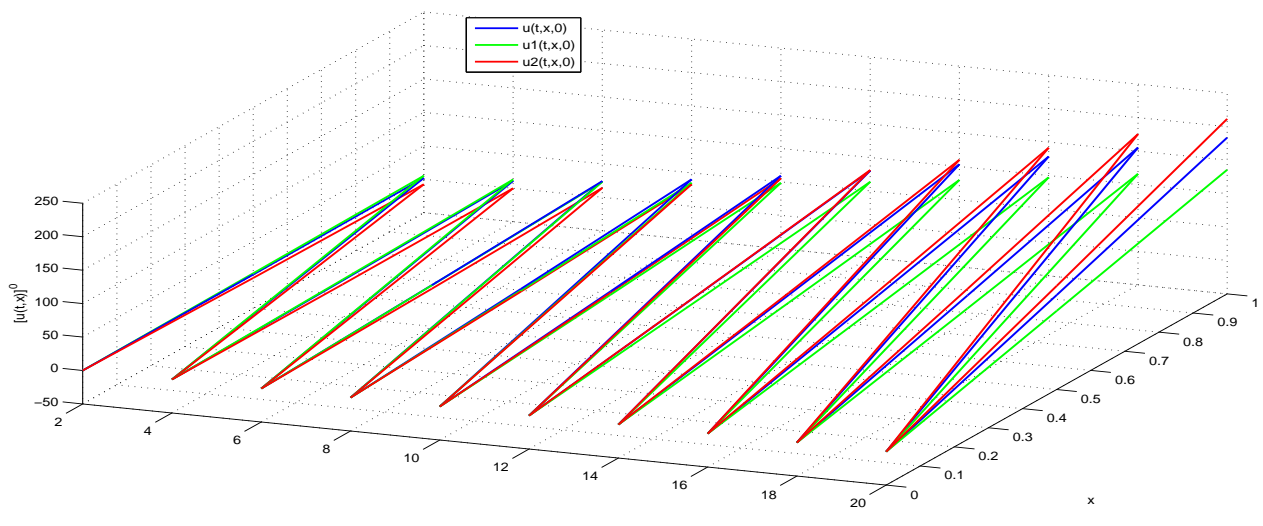

$[U(t, x)]^{0}$

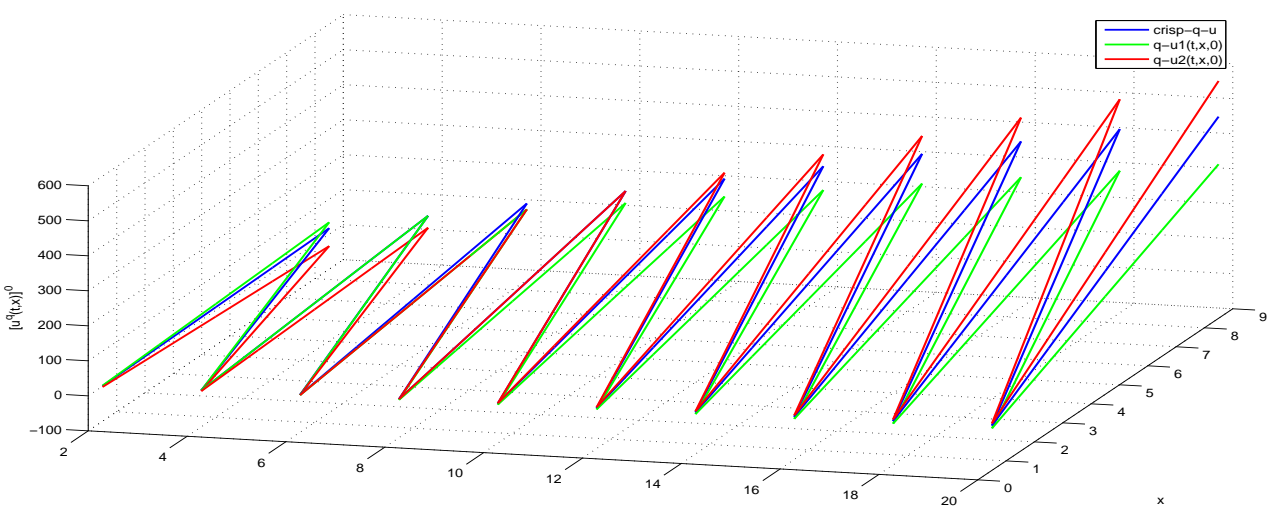

$\left[D_{t}^{q} U(t, x)\right]^{0}$

Figure 2. Fuzzy solution for $\alpha=0, q=0.55$ to 4.5

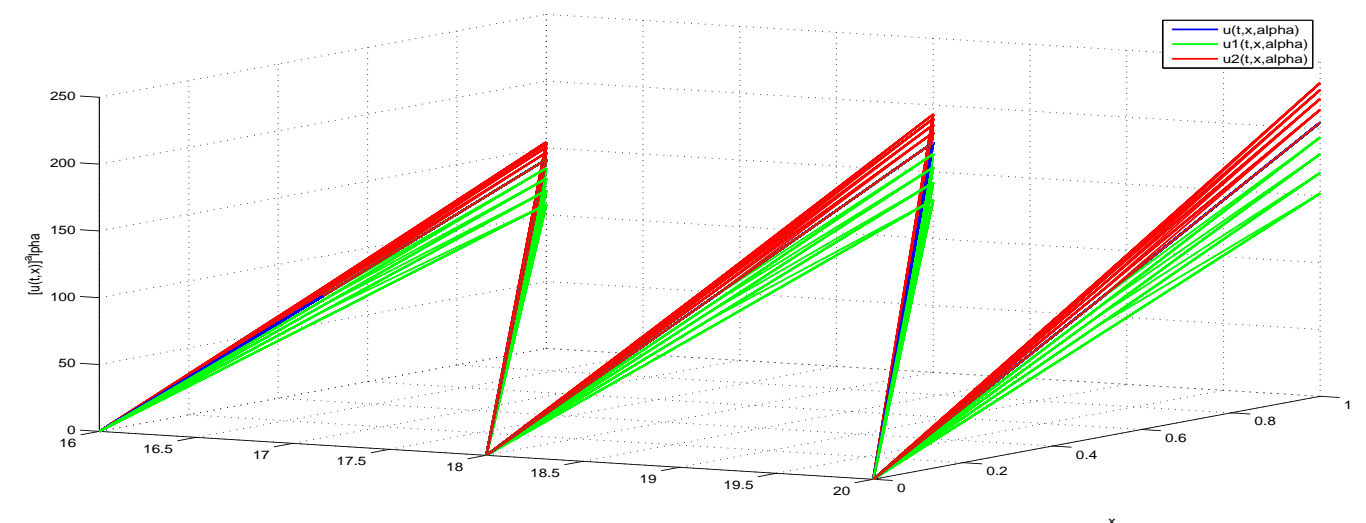

Figure 3. Fuzzy solution for $\alpha \in[0,1], q=0.55$ to 4.5

hence represent a fuzzy number for $x>0, t \geq-4$ for $\alpha \in[0,1]$.

Therefore 4.9 is a fuzzy solution for (4.4) which is given in figure 4 for $\alpha=0$ and in figure 5 for $\alpha \in[0,1]$. 


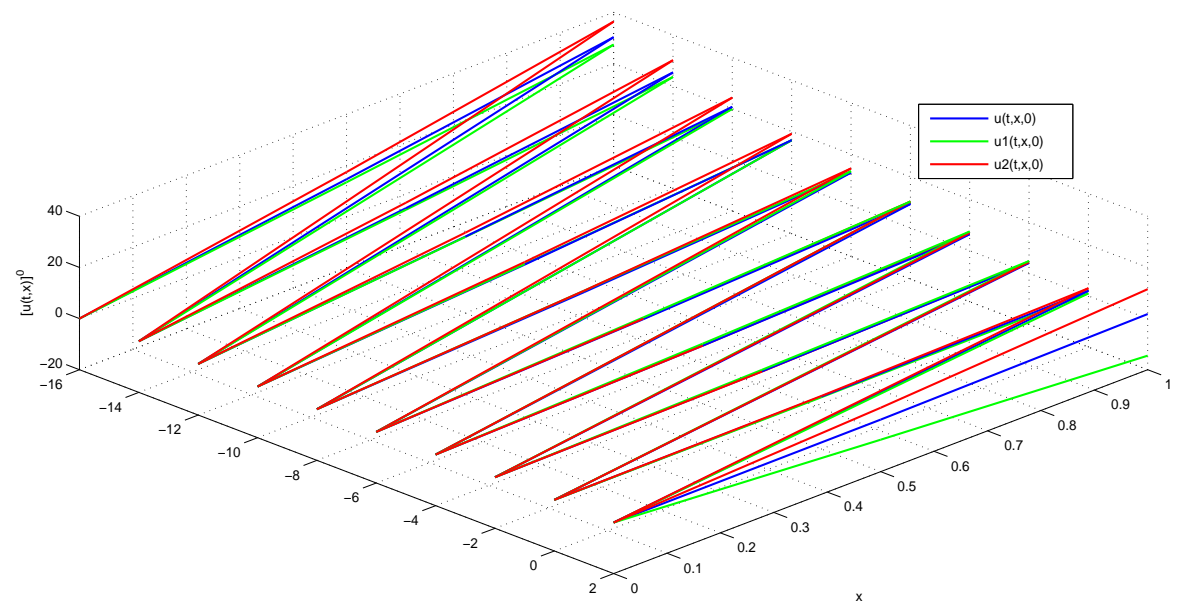

$$
[U(t, x)]^{0}
$$

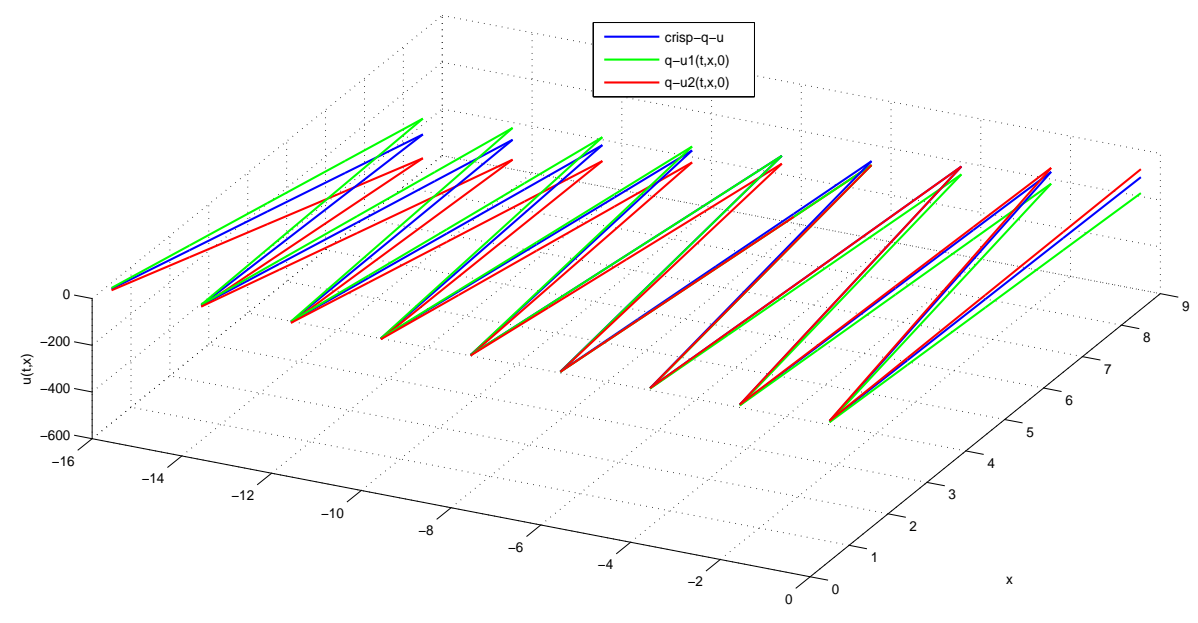

$\left[D_{t}^{q} U(t, x)\right]^{0}$

Figure 4. Fuzzy solution for $\alpha=0, q=0.55$ to 4.5

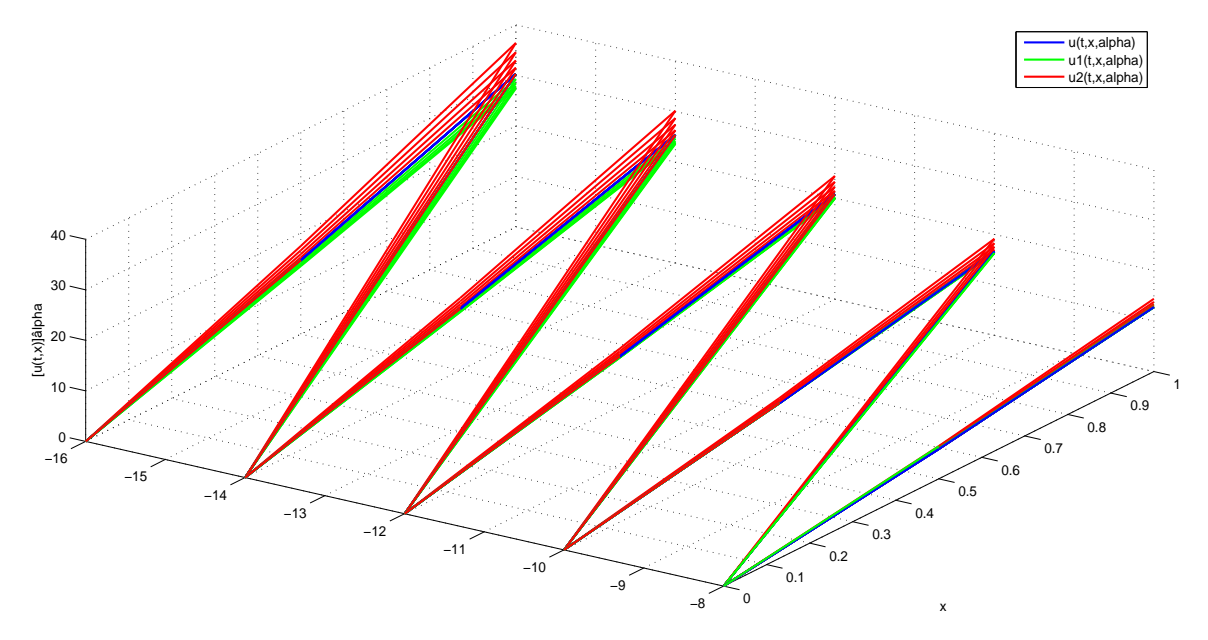

Figure 5. Fuzzy solution for $\alpha \in[0,1], \quad \mathrm{q}=0.55$ to 4.5 


\section{Conclusion}

Variational iteration method has been known as a powerful tool for solving many functional equations such as ordinary, partial differential equations, integral equations and fractional differential equations. In this article, if the crisp problem is not solvable explicitly we can not determine the mentioned domains precisely then by Variational iteration method (VIM), we obtain the solution of fuzzy fractional differential equation. We propose a new algorithm based on analysis of crisp solution. We establish a synthesis of crisp solution of fuzzy fractional differential equation, the definition of the Caputo fuzzy fractional derivatives [3] and the method proposed in [17. to solve fuzzy fractional differential equation. Finally the obtained solution are show graphically.

\section{RÉFÉRENCES}

[1] S.Arshad, V.Lupulescu. Fractional differential equation with the fuzzy initial conditions. Elect J Differ Equat $3420111-8$.

[2] S. Arshad, V. Lupulescu, On the fractional differential equations with uncertainty, Nonlinear Analysis 74, 2011 3685-3693

[3] A. Harir, S. Melliani and L. S. Chadli. Fuzzy fractional evolution equations and fuzzy solution operators, Advances in Fuzzy Systems, volume 2019, Article ID 5734190, 10 pages, 2019. https ://doi.org/10.1155/2019/5734190.

[4] A. Harir, S. Melliani and L. S. Chadli. Fuzzy generalized conformable fractional derivative. Advances in Fuzzy Systems, Advances in Fuzzy Systems, volume 2020, Article ID 1954975, 7 pages, 2019. https ://doi.org/10.1155/2020/1954975.

[5] L. S. Chadli, A. Harir and S. Melliani, Fuzzy Euler differential equation, SOP Transactions on Applied Mathematics, volume 2, number 1, January 2015.

[6] L. S. Chadli, A. Harir and S. Melliani, Solutions of fuzzy heat-like equations by variational iterative method , Annals of Fuzzy Mathematics and Informatics, Volume 10, number 1, 2015, pp 29-44.

[7] L. S. Chadli, A. Harir and S. Melliani, Solutions of fuzzy wave-like equations by variational iteration method. International Annals of Fuzzy Mathematics and Informatics, volume 8, number 4, 2014, 527-547.

[8] P. Diamond, P.E. Kloeden, Metric Spaces of Fuzzy Sets, Theory and Applications, World Scienific, Singapore, 1994.

[9] B.Dumitru, Machado Jos Ã (C) Antnio Tenreiro, Luo Albert CJ. Fract Dynam Control 2011.

[10] R. Getschel, W.Voxman, Elementary fuzzy calculus, Fuzzy sets and Systems 181986 31-43.

[11] Guo-cheng Wu, E.W.M. Lee, Fractional variational iteration method and its application, Physics Letters A $37420102506-2509$

[12] J.H. He, Variational iteration method-a kind of non-linear analytical technique : some examples, Int. J. Non-Linear Mech. 34 $1999699-708$.

[13] J.H. He, G.C. Wu, F. Austin, The variational iteration method which should be followed, Nonl. Sci. Lett. A 12010 1-30.

[14] J. H. He, Some asymptotic methods for strongly nonlinear equations, Int. J. Mod. Phys., B $2020061141-1199$.

[15] J.H. He, Approximate analytical solution for seepage flow with fractional derivatives in porous media, Comput. Methods Appl. Mech. Eng. 1671998 57-68.

[16] G. Jumarie, Table of some basic fractional calculus formulae derived from a modified Riemann-Liouville derivative for nondifferentiable functions, Appl. Math. Lett. 22 , 2009 378-385.

[17] O. Kaleva. Fuzzy differential equations. Fuzzy Sets and Systems, 24, 1987 301-317.

[18] M. Ma, M, Friedman, A. Kandel, A new fuzzy arithmetic, Fuzzy Sets and Systems 108, 1999 83-90.

[19] Meral FC, Royston TJ, Magi R. Fractional calculus in viscoelasticity, an experimental study. Commun Nonlinear Sci Numer Simulat $152010939-45$

[20] AM. Wazwaz, The variational iteration method for solving linear and nonlinear systems of PDEs, Comput. Math. Appl. 54 2007 95-902. 
(A.HARIR) Laboratory of Applied Mathematics and Scientific Computing, Sultan Moulay Slimane University, P.O. Box 523, Beni Mellal, 23000

Current address: Laboratory of Applied Mathematics and Scientific Computing, Sultan Moulay Slimane University, P.O. Box 523, Beni Mellal, 23000

E-mail address, A.Harir: atimad.harir@gmail.com

(S. Melliani) Laboratory of Applied Mathematics and Scientific Computing, Sultan Moulay Slimane University, P.O. Box 523, Beni Mellal, 23000

E-mail address, S. Melliani: s.melliani@usms.ma

(L.S.Chadli) Laboratory of Applied Mathematics and Scientific Computing, Sultan Moulay Slimane University, P.O. Box 523, Beni Mellal, 23000

E-mail address, L.S.Chadli: sa.chadli@yahoo.fr 